\title{
PRODUCTS OF POINCARÉ DOMAINS
}

\author{
ALEXANDER STANOYEVITCH
}

(Communicated by Palle E. T. Jorgensen)

\begin{abstract}
A domain $\Omega \subseteq \mathbb{R}^{N}$ of finite $N$-dimensional Lebesgue measure is a $p$-Poincare domain $(1 \leq p \leq \infty)$ if there exists a positive constant $K$ such that the $p$-Poincare inequality $\|u\|_{L^{p}(\Omega)} \leq K\|\nabla u\|_{L^{p}(\Omega)}$ is valid for all Sobolev functions $u \in W^{1, p}(\Omega)$ that integrate to zero. Define $K_{p}(\Omega)$ to be the smallest such $K$ if $\Omega$ is a $p$-Poincaré domain and to be infinity otherwise. We obtain comparability relations between $K_{p}\left(\Omega_{1} \times \Omega_{2}\right)$ and the pair $K_{p}\left(\Omega_{1}\right), K_{p}\left(\Omega_{2}\right)$. In particular, our results show that $p$-Poincare domains are closed under cartesian products (for all $p$ ), and that in case $p$ equals 2 , we have $K_{2}\left(\Omega_{1} \times \Omega_{2}\right)=\max \left\{K_{2}\left(\Omega_{1}\right), K_{2}\left(\Omega_{2}\right)\right\}$.
\end{abstract}

\section{INTRODUCTION}

Throughout this paper a "domain" $\Omega \subseteq \mathbb{R}^{N}$ will be an open connected set having finite $N$-dimensional Lebesgue measure, which we denote by $|\Omega|$. All "functions" are assumed to be real-valued and measurable. For an integrable function $h$ on $\Omega$ we let $h_{\Omega}$ denote its average value, i.e.,

$$
h_{\Omega}=\frac{1}{|\Omega|} \int_{\Omega} h(x) d x \text {. }
$$

For convenience of establishing some notation, we include the definition of the Sobolev spaces. More information on the Sobolev spaces and their functions can be found in [Z; GT, Chapter 7; Maz; A].

Definition. For $u, v \in L_{\mathrm{loc}}^{1}(\Omega)$ (meaning that $u$ and $v$ are functions on $\Omega$ that are integrable on compact subsets) and $1 \leq i \leq N$, we say that $v$ is the $i$ th weak partial derivative of $u$ on $\Omega$ (written as: $v=D^{i} u$ ) provided that the integration by parts formula

$$
\int_{\Omega} v(x) \varphi(x) d x=-\int_{\Omega} u(x) \frac{\partial}{\partial x_{i}} \varphi(x) d x
$$

is valid for each $\varphi \in \mathscr{C}_{0}^{1}(\Omega)$, the class of continuously differentiable functions having compact support in $\Omega$. If a weak derivative exists, it follows from

Received by the editors August 30, 1990 and, in revised form, May 3, 1991.

1980 Mathematics Subject Classification (1985 Revision). Primary 46E35; Secondary 47A10.

Key words and phrases. Poincaré domain, Poincaré inequality, Sobolev space, Neumann Laplacian.

The results of this paper come from the author's doctoral dissertation [Sta] done at the University of Michigan under the direction of Peter L. Duren and Allen L. Shields. 
the Lebesgue differentiation theorem that it is uniquely determined almost everywhere. If $D^{1} u, \ldots, D^{N} u$ all exist, we write $\nabla u$ for the weak gradient $\left(D^{1} u, \ldots, D^{N} u\right)$ of $u$.

For a number $p, 1 \leq p \leq \infty$, we define the (first order) Sobolev space

$$
W^{1, p}(\Omega) \equiv\left\{u \in L^{p}(\Omega): \nabla u \in L^{p}(\Omega)\right\} .
$$

We are now prepared to define Poincaré domains, the principal objects under investigation.

Definition. The best $p$-Poincaré constant $(1 \leq p \leq \infty)$ of $\Omega$ is given by

$$
K_{p}(\Omega)=\sup \left\{\frac{\left\|u-u_{\Omega}\right\|_{L^{p}(\Omega)}}{\|\nabla u\|_{L^{p}(\Omega)}}: u \in W^{1, p}(\Omega), u \neq \text { constant }\right\} .
$$

The domain $\Omega$ is said to be a p-Poincaré domain if $K_{p}(\Omega)$ is finite. Thus $p$-Poincaré domains are those domains for which there exists a positive number $K$ such that the $p$-Poincaré inequality

$$
\left\|u-u_{\Omega}\right\|_{L^{p}(\Omega)} \leq K\|\nabla u\|_{L^{p}(\Omega)}
$$

is valid for all $u \in W^{1, p}(\Omega)$.

The Poincaré inequalities are prototypes for general Sobolev inequalities that are important in the theories of partial differential equations and mathematical physics. The problem of estimating the best $p$-Poincaré constants and in particular, of classifying (geometrically) the $p$-Poincare domains has recently received a great deal of attention, see, e.g., [SmSt, Mar, SmSt, EH, J].

The purpose of this paper is to analyze the relationship between the best $p$ Poincare constants of the cartesian product of a pair of domains and those of the individual factor domains. The formula

$$
K_{p}\left(\Omega_{1} \times \Omega_{2}\right) \geq \max \left\{K_{p}\left(\Omega_{1}\right), K_{p}\left(\Omega_{2}\right)\right\}
$$

results from the fact that for each function $u(x) \in W^{1, p}\left(\Omega_{1}\right)$, the function $\tilde{u}(x, y) \equiv u(x)$ is in $W^{1, p}\left(\Omega_{1} \times \Omega_{2}\right)$ and the quotients appearing in (1) that correspond to these two functions are the same. Formula (3) implies that the cartesian product of a non- $p$-Poincaré domain with any domain whatsoever is again a non- $p$-Poincaré domain. A natural question thus arises: is the cartesian product of a pair of $p$-Poincaré domains again a $p$-Poincaré domain? Our main result is the following theorem that, in particular, yields an affirmative answer to this question.

Theorem 1.1. Suppose that $N_{1}$ and $N_{2}$ are positive integers. For domains $\Omega_{1} \subseteq$ $\mathbb{R}^{N_{i}}$ we have

$$
K_{p}\left(\Omega_{1} \times \Omega_{2}\right) \leq \begin{cases}2^{(p-1) / p} \sqrt[p]{K_{p}\left(\Omega_{1}\right)^{p}+K_{p}\left(\Omega_{2}\right)^{p}} & {[p<\infty]} \\ K_{\infty}\left(\Omega_{1}\right)+K_{\infty}\left(\Omega_{2}\right) & {[p=\infty]}\end{cases}
$$

and

$$
K_{2}\left(\Omega_{1} \times \Omega_{2}\right)=\max \left\{K_{2}\left(\Omega_{1}\right), K_{2}\left(\Omega_{2}\right)\right\} .
$$

Our proof of (4) is based on real variable methods and will be given in $\S 3$. It is possible to give a similar proof of (5). Instead of this, however, we give in $\S 4$ a proof of (5) that is based on a useful connection with the spectral theory of unbounded operators. In $\S 2$ we prove some lemmas that will be needed in $\S 3$. 
The paper concludes with $\S 5$ in which we discuss the problem of sharpening the estimate (4) and present some open questions.

\section{Preliminary lemmas}

We begin with a rather technical separability result which we use only in this section.

Lemma 2.1. Let $\Omega \subseteq \mathbb{R}^{N}$ be a domain, and let $j$ be an integer, $1 \leq j \leq N$. There exists a countable subset $D_{j} \subseteq \mathscr{C}_{0}^{1}(\Omega)$ such that to any $\varphi \in \mathscr{C}_{0}^{1}(\Omega)$, there corresponds a sequence $\left\langle\varphi_{n}\right\rangle \subseteq D_{j}$ with

$$
\int \varphi f=\lim \int \varphi_{n} f \text { and } \int\left(\frac{\partial}{\partial x_{j}} \varphi\right) f=\lim \int\left(\frac{\partial}{\partial x_{j}} \varphi_{n}\right) f
$$

for each $f \in L^{1}(\Omega)$

Proof. We begin by exhausting $\Omega$ by an increasing sequence of compact subdomains $\left\langle\Omega_{k}\right\rangle_{k=1}^{\infty}$ with the further property that $\bar{\Omega}_{k} \subseteq \Omega_{k+1}$ for each $k$. Let $\psi_{k}: \Omega \rightarrow[0,1]$ be a function in $\mathscr{C}_{0}^{1}(\Omega)$ that is supported in $\Omega_{k+1}$ and is identically equal to 1 on $\Omega_{k}$ (see, e.g., $[\mathrm{H}, \mathrm{p} .4]$ ).

The support of each function $\varphi \in \mathscr{C}_{0}^{1}(\Omega)$ will eventually be contained in some $\Omega_{k}$. It follows from the Stone-Weierstrass theorem that the polynomials in $\mathbb{Q}\left[x_{1}, x_{2}, \ldots, x_{n}\right]$, i.e., those with rational coefficients, are uniformly dense in $\mathscr{\mathscr { C }}\left(\overline{\Omega_{k+1}}\right)$. Thus for each $\varepsilon>0$, there exists a polynomial $\psi \in$ $\mathbb{Q}\left[x_{1}, x_{2}, \ldots, x_{n}\right]$ such that

$$
\frac{\sup }{\Omega_{k+1}}\left|\frac{\partial}{\partial x_{j}} \varphi-\psi\right| \leq \frac{\varepsilon}{1+\operatorname{diam}\left(\overline{\Omega_{K+1}}\right)}
$$

The same inequality persists if we replace $\psi$ by $\psi_{k} \psi$. Now consider the function

$$
\widetilde{\psi}: \mathbb{R}^{N} \rightarrow \mathbb{R}^{1}
$$

defined by

$$
\widetilde{\psi}\left(x_{1}, \ldots, x_{n}\right)=\int_{-\infty}^{x_{j}}\left(\psi_{k} \psi\right)\left(x_{1}, \ldots, x_{j-1}, t, x_{j+1}, \ldots, x_{n}\right) d t .
$$

Since the support of $\psi_{k} \psi$ lies in $\Omega_{k+1}$, we infer from (6) that

$$
\frac{\sup }{\Omega_{k+1}}|\varphi-\widetilde{\psi}| \leq \varepsilon \quad \text { and } \quad \frac{\sup }{\Omega_{k+1}}\left|\frac{\partial}{\partial x_{j}} \varphi-\frac{\partial}{\partial x_{j}} \widetilde{\psi}\right| \leq \varepsilon .
$$

The function $\widehat{\psi}=\psi_{k} \widetilde{\psi}$ satisfies the above inequalities with $C(k) \varepsilon$ in place of $\varepsilon$ and has the further property that it is in $\mathscr{C}_{0}^{1}(\Omega)$. Consequently, for a fixed $f \in L^{1}(\Omega)$, we have that

$$
\left|\int(\varphi-\widehat{\psi}) f\right| \leq \frac{\sup }{\Omega_{k+1}}|\varphi-\widehat{\psi}|\|f\|_{L^{1}\left(\Omega_{k+1}\right)} \leq(\text { CONSTANT }) \varepsilon,
$$

where the CONSTANT depends only on $k$ (and $f$ ) whereas $\varepsilon$ can be chosen arbitrarily small. We can obtain a similar upper bound for

$$
\left|\int \frac{\partial}{\partial x_{j}}(\varphi-\widehat{\psi}) f\right| \text {. }
$$


The lemma is now proved by letting $D_{j}$ be the countable set of all functions $\widehat{\psi}$ obtained as above.

The next two lemmas give ways to construct new Sobolev functions from old ones. The first lemma asserts that almost every slice of a Sobolev function is a Sobolev function; the latter shows that averages of Sobolev functions are Sobolev functions.

Lemma 2.2. Suppose that $1 \leq p \leq \infty$, that $\Omega_{i} \subseteq \mathbb{R}^{N_{i}}(i=1,2)$ are domains, and that $u \in W^{1, p}\left(\Omega_{1} \times \Omega_{2}\right)$. Then for almost every $x \in \Omega_{1}$, the function

$$
u_{x}: \Omega_{2} \rightarrow \mathbb{R}^{1}:: y \mapsto u(x, y)
$$

lies in $W^{1, p}\left(\Omega_{2}\right)$ and moreover, for $1 \leq j \leq N_{2}$ we have

$$
D^{j}\left(u_{x}\right)=\left(D_{\Omega_{2}}^{j} u\right)_{x} .
$$

Proof. Fubini's theorem implies that for almost every $x \in \Omega_{1}$, the functions $u_{x}$ and $\left(D_{\Omega_{2}}^{j} u\right)_{x}$ are in $L^{p}\left(\Omega_{2}\right)$. Thus, we need only check that (7) is valid for almost every $x \in \Omega_{1}$. Then

We fix an index $j, 1 \leq j \leq N_{2}$, and let $\psi \in \mathscr{C}_{0}^{1}\left(\Omega_{1}\right)$ and $\varphi \in \mathscr{C}_{0}^{1}\left(\Omega_{2}\right)$.

$$
\Phi(x, y) \equiv \psi(x) \varphi(y) \in \mathscr{C}_{0}^{1}\left(\Omega_{1} \times \Omega_{2}\right),
$$

and using Fubini's theorem we obtain

$$
\begin{gathered}
\int_{\Omega_{1}} \psi(x)\left[\int_{\Omega_{2}} \frac{\partial}{\partial y_{j}} \varphi(y) u_{x}(y) d y\right] d x=\int_{\Omega}\left(D_{\Omega_{2}}^{j} \Phi\right) u=-\int_{\Omega} \Phi\left(D_{\Omega_{2}}^{j} u\right) \\
=\int_{\Omega_{1}} \psi(x)\left[-\int_{\Omega_{2}} \varphi(y)\left(D_{\Omega_{2}}^{j} u\right)_{x}(y) d y\right] d x .
\end{gathered}
$$

Thus the bracketed functions of $x$ appearing in the first and last of the above integrals must be equal in the sense of distributions on $\Omega_{1}$ as $\psi$ is allowed to range over all $\mathscr{C}_{0}^{1}\left(\Omega_{1}\right)$ functions. However, Fubini's theorem implies that the two bracketed functions are both locally integrable on $\Omega_{1}$. Consequently, to each $\varphi \in \mathscr{C}_{0}^{1}\left(\Omega_{2}\right)$ there corresponds a set $G_{\varphi} \subseteq \Omega_{1}$ of full measure on which the bracketed functions agree, i.e., $x \in G_{\varphi}$ implies

$$
\int_{\Omega_{2}} \frac{\partial}{\partial y_{j}} \varphi(y) u_{x}(y) d y=-\int_{\Omega_{2}} \varphi(y)\left(D_{\Omega_{2}}^{j} u\right)(y) d y .
$$

Next, we let $D_{j}$ be a countable subset of $\mathscr{C}_{0}^{1}\left(\Omega_{2}\right)$ as in Lemma 2.1. Set $G=\bigcap_{\psi \in D_{j}} G_{\psi}$. It follows that (8) holds uniformly for every $x \in G$ and every $\varphi \in \mathscr{C}_{0}^{1}\left(\Omega_{2}\right)$. Put differently, this says that for each $x \in G$, the identity (7) is valid.

Lemma 2.3. Suppose that $\Omega_{i} \subseteq \mathbb{R}^{N_{1}}(i=1,2)$ are domains and that $u \in$ $W^{1, p}(\Omega)$, where $\Omega=\Omega_{1} \times \Omega_{2}$ and $1 \leq p \leq \infty$. Then the function

$$
U_{1}: \Omega_{1} \rightarrow \mathbb{R}^{1}
$$

defined by

$$
x \mapsto\left(u_{x}\right)_{\Omega_{2}} \equiv \frac{1}{\left|\Omega_{2}\right|} \int_{\Omega_{2}} u(x, y) d y
$$


lies in $W^{1, p}(\Omega)$, and for each $j\left(i \leq j \leq N_{1}\right)$ we have

$$
D^{j} U_{1}(x)=\frac{1}{\left|\Omega_{2}\right|} \int_{\Omega_{2}} D_{\Omega_{1}}^{j} u(x, y) d y .
$$

The proof of Lemma 2.3 is similar to that of Lemma 2.2; we omit it. The final result that we need here is an inequality that can be viewed as a vector-valued analogue of the familiar fact (for scalar valued functions) that the $L^{p}$-norm of a function dominates the absolute value of the integral of the function, when $p \geq 1$.

Lemma 2.4. Let $\langle X, \mathbb{S}, \mu\rangle$ be a probability space, $f_{j} \in L^{1}(\mu)(1 \leq j \leq N)$, and let $1 \leq p<\infty$. Then

$$
\left[\sum_{j=1}^{N}\left|\int_{X} f_{j}(x) d \mu(x)\right|^{2}\right]^{p / 2} \leq \int_{X}\left[\sum_{j=1}^{N}\left|f_{j}(x)\right|^{2}\right]^{p / 2} d \mu(x) .
$$

Lemma 2.4 is a special case of the vector-valued Jensen's inequality, which can be stated as follows (see [N, p. 55] for a proof). Suppose $D \subseteq \mathbb{R}^{n}$ is a convex domain and $\Phi: D \rightarrow \mathbb{R}$ is a convex function. Then for any probability space $\langle X, \mathbb{S}, \mu\rangle$ and vector-valued function $\vec{F}: X \rightarrow D$ we have

$$
\Phi\left(\int_{X} \vec{F}(x) d \mu(x)\right) \leq \int_{X} \Phi(\vec{F}(x)) d \mu(x) .
$$

Lemma 2.4 is then just this result with $\Phi: \mathbb{R}^{n} \rightarrow \mathbb{R}$ defined by $\Phi(x)=|x|^{p}=$ $\left(\sum_{i=1}^{n} x_{i}^{2}\right)^{p / 2}$.

\section{Proof of the general inequality}

Using the notation and results of $\S 2$, we proceed to give a proof of the estimate (4) of Theorem 1.1.

Case 1. $p<\infty$. Let $u \in W^{1, p}(\Omega)$. Fubini's theorem gives us that

$$
\int_{\Omega}\left|u-u_{\Omega}\right|^{p}=\int_{\Omega_{1}} \int_{\Omega_{2}}\left|u(x, y)-u_{\Omega}\right|^{p} d y d x .
$$

We invoke the elementary inequality: $(a+b)^{p} \leq 2^{p-1}\left(a^{p}+b^{p}\right)$, which is valid for $1 \leq p<\infty$ and $a, b \geq 0$, to conclude that the above integral is

$$
\leq \int_{\Omega_{1}} \int_{\Omega_{2}} 2^{p-1}\left[\left|u_{x}(y)-\left(u_{x}\right)_{\Omega_{2}}\right|^{p}+\left|\left(u_{x}\right)_{\Omega_{2}}-u_{\Omega}\right|^{p}\right] d y d x .
$$

Lemma 2.2 permits us to estimate this integral as

$$
\leq 2^{p-1} \int_{\Omega_{1}}\left[K_{p}\left(\Omega_{2}\right)^{p} \int_{\Omega_{2}}\left|\nabla u_{x}\right|^{p} d y+\left|\Omega_{2}\right|\left|U_{1}(x)-u_{\Omega}\right|^{p}\right] d x .
$$

We infer from Lemma 2.2 that $\left|\nabla u_{x}(y)\right| \leq|\nabla u(x, y)|$. Using this inequality in conjunction with Lemma 2.3, we can estimate the preceding integral as

$$
\leq 2^{p-1}\left[K_{p}\left(\Omega_{2}\right)^{p} \int_{\Omega}|\nabla u|^{p}+\left|\Omega_{2}\right| K_{p}\left(\Omega_{1}\right)^{p} \int_{\Omega_{1}}\left|\nabla U_{1}\right|^{p} d x\right] .
$$


Finally, in light of the formula for $\nabla U_{1}$ resulting from (9), we can invoke Lemma 2.4 to estimate the last integral as

$$
\leq 2^{p-1}\left[K_{p}\left(\Omega_{1}\right)^{p}+K_{p}\left(\Omega_{2}\right)^{p}\right] \int_{\Omega}|\nabla u|^{p} .
$$

Case 2. $p=\infty$. A few modifications in the above proof of Case 1 yield a proof that works here as well.

\section{Spectral theORy approach to the Case $p=2$}

Here we prove the identity (5) of Theorem 1.1. For clarity we shall first extract from the proof several preliminary results. Throughout this section we will assume familiarity with the standard notations and basic principles of the theory of unbounded operators, as is done, for example, in [K] or in [RS1, 2].

For a domain $\Omega \subseteq \mathbb{R}^{N}$, we let $q$ denote the gradient (quadratic) form on $\Omega$ with domain $D(q)=W^{1,2}(\Omega)$, i.e.,

$$
q(\varphi, \psi)=(\nabla \varphi, \nabla \psi) \quad \text { for } \varphi, \psi \in W^{1,2}(\Omega),
$$

where $(\cdot, \cdot)$ is the usual inner product on $\mathbb{R}^{N}$. The corresponding Friedrichs extension operator is then called the Neumann Laplacian (written as $-\Delta_{N}, \Omega$ or $-\Delta$ for short). The Neumann Laplacian is intimately connected with the 2-Poincare inequality on $\Omega$ as is shown by the following result.

Proposition 4.1. A domain $\Omega \subseteq \mathbb{R}^{N}$ is a 2-Poincaré domain if and only if there is a gap in the spectrum of the Neumann Laplacian $-\Delta_{N}, \Omega$ between zero and the positive elements of the spectrum. In any case, the infimum of the positive part of the spectrum in $\sigma\left(-\Delta_{N}, \Omega\right)$ coincides with the square of reciprocal of the best 2-Poincaré constant of $\Omega, K_{2}(\Omega)$.

I am very grateful to the referee for pointing out to me $\S 10$ of [DL] as the original source for this beautiful and useful connection. Some comments are in order. The Laplace operator used in this paper coincides with the Neumann Laplacian. This is easily seen in one direction by the uniqueness property of Friedrichs extensions and in the other direction by a simple integration by parts. Also, the result is only actually stated and proved under the supplementary assumption that the inclusion operator $W^{1,2}(\Omega) \hookrightarrow L^{2}(\Omega)$ is compact.

This assumption was only used in the application of the minimax principle to locate the bottom of the positive spectra of $-\Delta$. The minimax principle is, however, valid for all selfadjoint operators (see [R2, p. 75ff], and also page 338 therein for an interesting history of this result). The minimax principle had been known in the compact case since 1910 but was only extended to the general selfadjoint case by Kato at about the time of that [DL] was written. In the present situation, the eigenvector $u(x) \equiv 1$ corresponds to the "ground state" of $-\Delta$. The minimax principle then tells us that the infimum of the positive part of $\sigma(-\Delta)$ is given by

$$
\sup _{\varphi} \inf _{\substack{\psi \in D(-\Delta) \\\|\psi\|=1 \\ \psi \perp \varphi}}(-\Delta \psi, \psi)=\inf _{\substack{\psi \in D(-\Delta) \\\|\psi\| 1 \\ \text { N } \|=1}}(-\Delta \psi, \psi) .
$$

Theorem 4.2. Let $\Omega_{1}$ and $\Omega_{2}$ be arbitrary domains in $\mathbb{R}^{N_{1}}$ and $\mathbb{R}^{N_{2}}$ respectively, and let $\Omega$ denote the product domain $\Omega_{1} \times \Omega_{2} \subseteq \mathbb{R}^{N_{1}+N_{2}}$. We denote the 
corresponding Neumann Laplacians by $-\Delta_{1},-\Delta_{2}$, and $-\Delta$, respectively. If we identify $L^{2}(\Omega)$ "naturally" with $L^{2}\left(\Omega_{1}\right) \otimes L^{2}\left(\Omega_{2}\right)$, we then have the identity,

$$
-\Delta=\overline{\left.\left(\left(-\Delta_{1}\right) \otimes I+I \otimes\left(-\Delta_{2}\right)\right)\right|_{D\left(-\Delta_{1}\right) \otimes D\left(-\Delta_{2}\right)}} .
$$

Proof. Let us use $R$ to denote the operator $\left(-\Delta_{1}\right) \otimes I+I \otimes\left(-\Delta_{2}\right)$ on the domain $D\left(-\Delta_{1}\right) \otimes D\left(-\Delta_{2}\right)$. It follows from Exercise VIII.47 of [R1] that $R$ is essentially selfadjoint, in other words, $\bar{R}$ is selfadjoint. Since $-\Delta$ is also selfadjoint, and selfadjoint operators are maximal symmetric operators (i.e., if $A$ is selfadjoint $B$ is symmetric and $A \subseteq B$ then $A=B$. Proof: $A=A^{*} \supseteq B^{*} \supseteq B$. $\square$ ) it suffices to show that these two operators agree on a typical tensor $\varphi \otimes \psi \epsilon$ $D\left(-\Delta_{1}\right) \otimes D\left(-\Delta_{2}\right)$.

By uniqueness of Friedrichs extensions, it is enough to show that $\varphi \otimes \psi$ $(=\varphi(x) \cdot \psi(y))$ lies in $D(q)\left(W^{1,2}(\Omega)\right)$ and for each $\Phi \in W^{1,2}(\Omega)$, we have $q(\varphi \otimes \psi, \Phi)=(R(\varphi \otimes \psi), \Phi)_{L^{2}(\Omega)}$.

Fubini's theorem clearly yields that $\varphi \otimes \psi \in D(q)$. We adopt the following notation: $\Phi_{x}(y)=\Phi(x, y)=\Phi_{y}(x)$, where $\Phi$ is any function on $\Omega$. The apparent ambiguity inherent in this notation is transcended by the fact that $x$ and $y$ will not be substituted by any other variables. Now let $\Phi \in W^{1,2}(\Omega)$. Recall that from Lemma 2.2, for a.e. $y \in \Omega_{2}, \Phi_{y} \in W^{1,2}\left(\Omega_{1}\right)$; and symmetrically, $\Phi_{x} \in W^{1,2}\left(\Omega_{2}\right)$ for a.e. $x \in \Omega_{1}$. By invoking the properties of Friedrichs extensions, we obtain

$$
\begin{aligned}
(R(\varphi & \otimes \psi), \Phi)_{L^{2}(\Omega)} \\
& =\left(-\Delta_{1} \varphi(x) \psi(y)-\varphi(x) \Delta_{2} \psi(y), \Phi(x, y)\right)_{L^{2}(\Omega)} \\
& =\int_{\Omega_{2}} \psi(y)\left(-\Delta_{1} \varphi, \Phi_{y}\right)_{L^{2}\left(\Omega_{1}\right)} d y+\int_{\Omega_{1}} \varphi(x)\left(-\Delta_{2} \psi, \Phi_{x}\right)_{L^{2}\left(\Omega_{2}\right)} d x \\
& =\int_{\Omega_{2}} \psi(y)\left(\nabla_{x} \varphi, \nabla_{x} \Phi_{y}\right)_{L^{2}\left(\Omega_{1}\right)} d y+\int_{\Omega_{1}} \varphi(x)\left(\nabla_{y} \psi, \nabla_{y} \Phi_{x}\right)_{L^{2}\left(\Omega_{2}\right)} d x \\
& =\int_{\Omega_{1}} \int_{\Omega_{2}}\left[\psi(y) \nabla_{x} \varphi(x) \cdot \nabla_{x} \Phi(x, y)+\varphi(x) \nabla_{y} \psi(y) \cdot \nabla_{y} \Phi(x, y)\right] d y d x \\
& =\iint_{\Omega_{1} \times \Omega_{2}} \nabla(\varphi \otimes \psi) \cdot \nabla \Phi=q(\varphi \otimes \psi, \Phi) .
\end{aligned}
$$

The proof is thus complete.

The following result, which is a special case of the corollary [RS1, p. 301], will be the final ingredient we will need for the proof of (5).

Proposition 4.3. If $A_{i}$ is a selfadjoint operator with domain $D\left(A_{i}\right) \subseteq \mathscr{H}_{i}(i=$ $1,2)$, then

$$
\sigma\left[\overline{\left.\left(A_{1} \otimes I+I \otimes A_{2}\right)\right|_{D\left(A_{1}\right) \otimes D\left(A_{2}\right)}}\right]=\overline{\sigma\left(A_{1}\right)+\sigma\left(A_{2}\right)} .
$$

Proof of (5). In this proof we shall be using the notations for the three Neumann Laplacians which were given in Theorem 4.2. Proposition 4.1 tells us that

$$
K_{2}\left(\Omega_{1} \times \Omega_{2}\right)=\sup \left\{\frac{1}{\lambda} \mid \lambda \in \sigma(-\Delta) \backslash\{0\}\right\}^{1 / 2} .
$$


By Theorem 4.2 and Proposition 4.3, we can rewrite this as

$$
\begin{aligned}
& =\sup \left\{\frac{1}{\lambda_{1}+\lambda_{2}} \mid \lambda_{i} \in \sigma\left(-\Delta_{i}\right) \text { not both zero }\right\}^{1 / 2} \\
& =\max \left\{K_{2}\left(\Omega_{1}\right), K_{2}\left(\Omega_{2}\right)\right\} .
\end{aligned}
$$

\section{CONCLUDING REMARKS AND QUESTIONS}

Theorem 1.1 suggests the following questions.

Question 5.1. For $1 \leq p \leq \infty, p \neq 2$, what is the smallest constant $\Lambda(p)$ for which

$$
K_{p}\left(\Omega_{1} \times \Omega_{2}\right) \leq \Lambda(p) \max \left\{K_{p}\left(\Omega_{1}\right), K_{p}\left(\Omega_{2}\right)\right\} ?
$$

We believe that $\Lambda(p)$ is realized in the simple case in which $\Omega_{1}=$ ] $-1,1[$ $=\Omega_{2}$. The author has calculated all of the best $p$-Poincaré constants of $]-1$, 1[ (see [Sta]), they are

$$
K_{p}(]-1,1[)= \begin{cases}1 & \text { if } p=1, \infty \\ \frac{p \sin (\pi / p)}{\pi(p-1)^{1 / p}} & \text { if } 1<p<\infty .\end{cases}
$$

The formula (5) makes it seem plausible that perhaps $\Lambda(p)$ equals 1 for all $p$. In case $p=\infty$, we can consider the function $f:]-1,1\left[^{2} \rightarrow \mathbb{R}^{1}\right.$ defined by

$$
(x, y) \mapsto y+x,
$$

to find that $\left.K_{\infty}(]-1,1{ }^{2}\right) \geq 2^{1 / 2}>1=K_{\infty}$ (] $-1,1[)$ so that (5) cannot be true for $K_{\infty}$ in place of $K_{2}$ (and in fact, by continuity of $L^{p}$ norms, it cannot be true for any sufficiently large $p$ ). At the other extreme, i.e., when $p=1$, the problem is quite a bit more recalcitrant

Question 5.2. Is $K_{1}(]-1,1\left[^{2}\right)$ larger than $1\left(=K_{1}(]-1,1[)\right)$ ?

\section{REFERENCES}

[A] R. A. Adams, Sobolev spaces, Academic Press, New York 1975.

[DL] J. Deny and J. L. Lions, Les espaces du type de Beppo Levi, Ann. Inst. Fourier (Grenoble) 5 (1953-54), 305-370.

[EH] W. D. Evans and D. J. Harris, Sobolev embeddings for generalized rigid domains, Proc. London Math. Soc. (3) 54 (1987), 141-175.

[GT] D. Gilbarg and N. S. Trudinger, Elliptic partial differential equations of second order, Springer-Verlag, New York, 1983.

[H] L. Hörmander, Linear partial differential operators, Springer-Verlag, Berlin and Heidelberg, 1963.

[J] D. S. Jerison, The Poincaré inequality for vector fields satisfying Hörmander's condition, Duke Math. J. 53 (1986), 503-523.

[K] T. Kato, Perturbation theory for linear operators, 2nd ed., Springer-Verlag, Berlin and Heidelberg, 1976.

[Mar] O. Martio, John domains, bilipschitz balls and the Poincaré inequality, Rev. Roumaine Math. Pures Appl. (1988), 107-112.

[Maz] V. G. Maz'ja, Sobolev spaces, Springer-Verlag, San Francisco, 1985.

[N] J. Neveu, Mathematical foundation of the calculus of probability, Holden-Day, 1965. 
[RS1] M. Reed and B. Simon, Methods of modern mathematical physics-vol. I: Functional analysis, Academic Press, New York, 1980.

[RS2] _ Methods of modern mathematical physics_vol. IV: Analysis of operators, Academic Press, New York, 1980.

[SmSt1] W. Smith and D. A. Stegenga, Poincaré domains in the plane, Complex Analysis, Joensuu-Proceedings of R. Nevalinna Colloquium (I. Laine, S. Rickman, and T. Sorvali, eds.), Lecture Notes in Math., vol. 1351, Springer-Verlag, Berlin and Heidelberg, 1987, pp. 312-327.

[SmSt2] _ Hölder domains and Poincaré domains, Trans. Amer. Math. Soc. 319 (1990), $67-100$.

[Sta] A. Stanoyevitch, Geometry of Poincaré domains, Doctoral Dissertation, Univ. of Michigan, 1990.

[Z] W. P. Ziemer, Weakly differentiable functions, Graduate Texts in Math., vol. 120, SpringerVerlag, New York, 1989.

Department of Mathematics, University of HawaiI, Honolulu, Hawail 96822 